\title{
Spectral Analysis of Microwave Background Perturbations Induced by Cosmic Strings
}

\author{
Leandros Perivolaropoulos*†
}

\begin{abstract}
Using a simple analytic model based on seed superposition we obtain the spectrum of microwave background perturbations induced by cosmic strings on all angular scales larger than about 2 armin. We assume standard recombination in an Einstein de Sitter universe with $h=1 / 2$ and study the fluctuation spectrum along a great circle in the sky. Doppler and potential perturbations on the last scattering surface (Sachs-Wolfe effect) are shown to dominate over post-recombination perturbations on scales smaller than about 2 degrees. Using a filter function corresponding to the COBE experiment we obtain an effective power spectrum index $n_{\text {eff }} \simeq 1.35$ in good agreement with the recently announced second year COBE data showing $n_{\text {eff }} \simeq 1.5$. The only free parameter $(G \mu)$ of the string model is fixed by normalizing on the COBE detection leading to $G \mu=1.6$. Other parameters (e.g. the rms string velocity) are fixed by comparing with string simulations. Using these parameter values we compare the rms temperature fluctuations $\left(\frac{\Delta T}{T}\right)_{r m s}$ predicted for ongoing experiments (Tenerife, SP91, MAX, OVRO etc) with detections and with the corresponding predictions of inflationary models.
\end{abstract}

Subject headings: cosmic microwave background - cosmic strings

${ }^{*}$ Division of Theoretical Astrophysics, Harvard-Smithsonian Center for Astrophysics 60 Garden St. Cambridge, Mass. 02138, USA.

${ }^{\dagger}$ also Visiting Scientist, Department of Physics Brown University Providence, R.I. 02912, U.S.A. 


\section{Introduction}

The rapid development of both observations and theory has turned the search for the origin of cosmic structure into one of the most exciting fields of scientific research. Succesful observations performed during the past few years have imposed severe constraints on theories of structure formation. In spite of these constraints one important question remains open: What is the origin of primordial fluctuations that gave rise to structure in the universe? Two classes of theories attempting to answer this question have emerged during the past ten years and have managed to survive through the observational constraints with only minor adjustments.

According to the first class, primordial fluctuations are produced by quantum fluctuations of a linearly coupled scalar field during a period of inflation (Hawking 1982; Starobinsky 1982; Guth \& Pi 1982; Bardeen, Steinhardt \& Turner 1983). These fluctuations are subsequently expected to become classical and provide the progenitors of structure in the universe. Because of the extremely small linear coupling of the scalar field, needed to preserve the observed large scale homogeneity, the inflationary perturbations are expected by the central limit theorem, to obey Gaussian statistics. This is not the case for the second class of theories.

According to the second class of theories (Kibble 1976; Vilenkin 1981; Vilenkin 1985; Turok 1989; Brandenberger 1992), primordial perturbations are provided by seeds of trapped energy density produced during symmetry breaking phase transitions in the early universe. Such symmetry breaking is predicted by Grand Unified Theories (GUT's) to occur at early times as the universe cools and expands. The geometry of the produced seeds, known as topological defects is determined by the topology of the vaccuum manifold of the physically realized GUT. Thus the defects may be pointlike (monopoles), linelike (cosmic strings), planar (domain walls) or collapsing pointlike (textures).

The cosmic string theory (Vilenkin 1981) for structure formation is the oldest and (together with textures (Turok 1989)) best studied theory of the topological defect class. By fixing its single free parameter $G \mu$ ( $\mu$ is the effective mass per unit length of the wiggly string and $G$ is Newtons constant) to a value consistent with microphysical requirements coming from GUT's, the theory may automatically account for large scale filaments and sheets (Vachaspati 1986; Stebbins et. al. 1987; Perivolaropoulos, Brandenberger \& Stebbins 1990; Vachaspati \& Vilenkin 1991; Vollick 1992; Hara \& Miyoshi 1993), galaxy formation at epochs $z \sim 2-3$ (Brandenberger et. 
al. 1987) and galactic magnetic fields (Vachaspati 1992b). It can also provide large scale peculiar velocities (Vachaspati T. 1992a; Perivolaropoulos \& Vachaspati 1993) and is consistent with the amplitude, spectral index (Bouchet, Bennett \& Stebbins 1988; Bennett, Stebbins \& Bouchet 1992; Perivolaropoulos 1993a; Hindmarsh 1993) and the statistics (Gott et. al. 1990; Perivolaropoulos 1993b; Moessner, Perivolaropoulos \& Brandenberger 1993; Coulson et. al. 1993; Luo 1993) of the cosmic microwave background (CMB) anisotropies measured by the COBE collaboration (Smoot et. al. 1992; Wright et. al. 1992) on large angular scales $\left(\theta \sim 10^{\circ}\right)$.

The CMB observations provide a valuable direct probe for identifying signatures of cosmic strings. There are three main mechanisms by which strings can produce CMB temperature fluctuations.

The first mechanism has been well studied both analytically (Brandenberger \& Turok 1986; Stebbins 1988; Veeraraghavan \& Stebbins 1990; Perivolaropoulos 1993a; Perivolaropoulos 1993b; Moessner et. al. 1993) and using numerical simulations (Bouchet et.al. 1988; Bennett et. al. 1992) and is known as the Kaiser-Stebbins effect (Kaiser \& Stebbins 1984; Gott 1985). According to this effect, moving long strings present between the time of recombination $t_{r e c}$ and the present time $t_{0}$, produce step-like temperature discontinuities between photons that reach the observer through opposite sides of the string. These discontinuities are due to the peculiar nature of the spacetime around a long string which even though is locally flat, globally has the geometry of a cone with deficit angle $8 \pi G \mu$. The magnitude of the discontinuity is proportional to the deficit angle, to the string velocity $v_{s}$ and depends on the relative orientation between the unit vector along the string $\hat{s}$ and the unit photon wave-vector $\hat{k}$. It is given by (Stebbins 1988)

$$
\frac{\delta T}{T}= \pm 4 \pi G \mu v_{s} \gamma_{s} \hat{k} \cdot\left(\hat{v}_{s} \times \hat{s}\right)
$$

where $\gamma_{s}$ is the relativistic Lorentz factor and the sign changes when the string is crossed. The angular scale over which this discontinuity persists is given by the radius of curvature of the string $\Psi(t)=\xi \Theta(t) / 2$ (the parameter $\xi$ measures the string curvature radius as a fraction of the horizon angular scale $\Theta(t))$.

The second mechanism is based on the Doppler effect. Moving long strings present on or before the last scattering surface produce, due to their deficit angle, velocity fields directed towards the surface swept by the string. These perturbations affect both the plasma and the dark matter. The growth of velocity perturbations produced before recombination on the 
plasma, is prevented by pressure and photon drag. However, velocity fields produced on the plasma last scatterers of photons by moving long strings at the time of recombination are important because they produce temperature fluctuations on the scattered photons via the Doppler effect. The magnitude of these fluctuations can be obtained from the velocity perturbations (Vachaspati \& Vilenkin 1991; Vachaspati 1992a) induced by a long string as

$$
\frac{\delta T}{T}=\hat{k} \cdot \vec{v}=\lambda \pi G \mu v_{s} \gamma_{s} \hat{k} \cdot\left(\hat{v}_{s} \times \hat{s}\right)
$$

where

$$
\lambda=\left(1+\frac{\left(1-\frac{T}{\mu}\right)}{2<\left(v_{s} \gamma_{s}\right)^{2}>}\right)
$$

and $\mathrm{T}$ is the tension of the wiggly long string estimated by simulations to be $T \simeq 0.7 \mu$. The scale over which these fluctuations persist is the radius of curvature of long strings at $t_{r e c}\left(\Psi\left(t_{r e c}\right)\right)$.

The third mechanism is based on potential fluctuations on the last scattering surface produced by both loops and long string wakes. Wakes are planar density enhancements induced by the growing velocity perturbations of long strings on dark matter (Stebbins et. al. 1987; Perivolaropoulos et. al. 1990). These perturbations begin to grow at about the time of equal matter and radiation $t_{e q}$ (assuming Cold Dark Matter (CDM)). A wake produced by a long string at time $t_{i}$ has angular dimensions $\Psi\left(t_{i}\right) \times v_{s} \gamma_{s} \Theta\left(t_{i}\right)$ and a surface density at time $t$ given by (Perivolaropoulos et. al. 1990)

$$
\sigma\left(t_{i}, t\right)=\lambda 4 \pi G \mu v_{s} \gamma_{s} t_{i} \rho\left(t_{i}\right)\left(\frac{t}{t_{i}}\right)^{2 / 3}
$$

where $\rho\left(t_{i}\right)$ is the mean dark matter density at $t_{i}$. The temperature perturbation due to the potential $\Phi\left(x, t_{r e c}\right)$ (Sachs \& Wolfe 1967; Stebbins 1993) produced by the wakes present on the last scattering surface is

$$
\frac{\delta T}{T}=\frac{1}{3} \Phi_{w}\left(x, t_{r e c}\right)=\frac{1}{3} 4 \pi G \sigma\left(t_{i}, t_{r e c}\right) h(x)
$$

where $h(x)=x \cos \phi$ is the perpendicular distance to the wake as a function of the distance $x$ from the wake on the last scattering surface and $\phi$ is the angle between the photon wave-vector and the wake surface. As in the previous cases, the angular distance from the wake over which this perturbation persists is approximatelly $\Psi\left(t_{i}\right)$.

Loops present on the last scattering surface with their accreted dark matter are also expected to contribute to the potential fluctuations. It will be 
seen however that their contribution is minimal due to their very small size indicated by numerical simulations. According to simulations (Bennett \& Bouchet 1988) loops are produced with relativistic velocities (which quickly erase any initial correlations) and typical size $R(t) \simeq 10^{-4} H^{-1}(t)\left(H^{-1}(t)\right.$ is the Hubble scale at time $t$ ), which for $t=t_{\text {rec }}$ corresponds to an angular scale of about 0.3 arcsec (even though the largest loops can be about ten times larger (Bouchet 1988)). This scale is too small for any observable effects in present experiments. For completeness however we will include loops in our analysis in order to explicitly demonstrate that they are unimportant compared to other types of perturbations.

The temperature fluctuations induced by a loop of radius $R$, due to the disturbance of the last scattering surface potential at a distance $x$ from the center of the loop may be approximated by

$$
\begin{array}{ll}
\frac{\delta T}{T}=\frac{1}{3} \Phi_{l}\left(x, t_{r e c}\right) \simeq \frac{\beta G \mu}{3}\left(\frac{t_{r e c}}{t_{i}}\right)^{2 / 3} \quad|x| \leq R \\
\frac{\delta T}{T} \simeq 0 \quad|x|>>R
\end{array}
$$

where $\beta$ is a parameter determining the length of string in a loop of radius $R$ (typically $\beta \leq 10$ ). The time dependent growth factor takes into account the accretion of CDM (as in the case of wakes).

\section{Derivation of Spectrum}

The above three mechanisms have specified four distinct types of seed functions that need to be superimposed (in different ways) in order to construct the CMB spectrum of string induced temperature fluctuations. The next step is to give an expression of the spectrum in terms of these seed functions. For simplicity we will focus on perturbations along a great circle on the sky. By isotropy, our results for the correlation function and the spectrum $P(k)$ are valid for any such circle and the extension of our results to patches will be shown to be straightforward.

Consider a seed function $f_{1}^{\Psi}(\theta)$ of size proportional to $\Psi$, superimposed at random positions $\theta_{n}$ and with variable amplitude $a_{n}, N$ times on a circle $(-\pi \leq \theta<\pi)$. The resulting function is

$$
f(\theta)=\sum_{n=1}^{N} a_{n} f_{1}^{\Psi}\left(\theta-\theta_{n}\right)=\frac{1}{2 \pi} \sum_{n=1}^{N} a_{n} \sum_{k=-\infty}^{+\infty} \tilde{f}_{1}^{\Psi}(k) e^{i k\left(\theta-\theta_{n}\right)}
$$


where

$$
\tilde{f}_{1}^{\Psi}(k) \equiv \int_{-\pi}^{+\pi} d \theta f_{1}^{\Psi}(\theta) e^{-i k \theta}
$$

is the Fourier transform of $f_{1}(\theta)$. The Fourier transform of $f(\theta)$ is

$$
\tilde{f}(k)=\tilde{f}_{1}^{\Psi}(k) \sum_{n=1}^{N} a_{n} e^{i k \theta_{n}}
$$

The probability distribution of the phases of this pattern has been studied previously (Perivolaropoulos 1993c). Here we focus on the power spectrum of the resulting pattern which is

$$
P_{0}(k) \equiv<|\tilde{f}(k)|^{2}>=N\left|\tilde{f}_{1}^{\Psi}(k)\right|^{2}<\left|a_{n}\right|^{2}>
$$

where $<>$ denotes ensemble average.

In a cosmological setup the role of seeds is played by topological defects which obey a 'scaling solution' and therefore their size is a fixed fraction of the horizon at any given time. As the comoving horizon expands by a factor $\alpha$, the size (total number) of superimposed seeds on the great circle will also increase (decrease $\rrbracket$ ) by the same factor. Therefore, after $Q$ expansion steps the resulting spectrum will be

$$
P_{Q}(k)=\sum_{q=0}^{Q} P_{q}(k) \equiv \sum_{q=0}^{Q} \frac{N}{\alpha^{q}}\left|\tilde{f}_{1}^{\alpha^{q} \Psi}(k)\right|^{2}<\left|a_{n}\right|^{2}>
$$

This is a general result that can be easily applied to any particular type of seed function $f_{1}(\theta)$ provided the following quantities are specified

1. The number of seeds $N$ at the first expansion step. For a scaling defect with M seeds per horizon scale we have $N\left(t_{i}\right)=M \times \frac{2 \pi}{\Theta\left(t_{i}\right)}$ where $\Theta\left(t_{i}\right)$ is the angular size of the horizon at the first expansion step. For $H_{0}=$ $50 \mathrm{~km} /(\mathrm{sec} \cdot \mathrm{Mpc})$, (i.e. $\mathrm{h}=1 / 2)$ and $\Omega_{0}=1$ we have $N\left(t_{\text {rec }}\right) \simeq 200 \mathrm{M}$ and $N\left(t_{e q}\right) \simeq 450 M$ since $\Theta\left(t_{r e c}\right)=1.8^{\circ}$ and $\Theta\left(t_{e q}\right)=0.8^{\circ}$.

2. The maximum and minimum size of superimposed seeds $\left(\Psi_{\min }\left(t_{i}\right), \Psi_{\max }\left(t_{i}\right)\right) \equiv\left(\xi \Theta\left(t_{i}\right) / 2, \xi \Theta\left(t_{f}\right) / 2\right)$. For initial and final times of superposition $\left(t_{i}, t_{f}\right)=\left(t_{r e c}, t_{0}\right)$ (to be used for the Kaiser-Stebbins effect) we have

$$
\left(\Psi_{\min }, \Psi_{\max }\right)=(0.016 \xi, \pi \xi)
$$

\footnotetext{
${ }^{1}$ since the total number of horizons on the circle decreases
} 
while for $\left(t_{i}, t_{f}\right)=\left(t_{e q}, t_{r e c}\right)$ (to be used for the scales of potential perturbations) we have

$$
\left(\Psi_{\min }, \Psi_{\max }\right)=(0.007 \xi, 0.016 \xi)
$$

The number of expansion steps is obtained simply as

$$
Q=\frac{\log \left(\frac{\Psi_{\max }}{\Psi_{\min }}\right)}{\log \alpha}
$$

3. The comoving horizon expansion factor $\alpha$. Taking each expansion step when the physical horizon scales by $\delta$ in size leads to $\alpha=\delta^{1 / 3}$. Increasing $\alpha$ tends to increase each term in the sum (12) but this effect is compensated by the decrease in $Q$ due to (15). Thus for reasonable values of $\delta(1.5 \leq \delta \leq 3)$ our results are rather insensitive to the value of $\alpha$. In what follows we use $\delta=2$ which implies $\alpha=1.26$.

4. The magnitude of $a_{n}$ and the form $f_{1}(\theta)$ of the seed functions given by equations (1), (2), (5) and (6).

We are now in position to determine the contribution to the power spectrum for each one of the three mechanisms by which strings can produce temperature fluctuations on the CMB.

1. Kaiser-Stebbins effect: It is easy to see from (1) that in this case

$$
\begin{array}{lcc}
f_{1}^{\Psi}(\theta)=-1 & 0 \leq \theta \leq \Psi \\
f_{1}^{\Psi}(\theta)=+1 & 0 \geq \theta \geq-\Psi \\
f_{1}^{\Psi}(\theta)=0 & \text { otherwise }
\end{array}
$$

while

$$
a_{n}=4 \pi G \mu\left(v_{s} \gamma_{s}\right)_{n} \cos \phi_{n}
$$

where $\cos \phi_{n}=\hat{k} \cdot\left(\hat{v}_{s} \times \hat{s}\right)_{n}$. It is now easy to show that

$$
\tilde{f}_{1}^{\Psi}(k)=\frac{4 \sin ^{2}(k \Psi / 2)}{k}
$$

and

$$
<\left|a_{n}\right|^{2}>=\frac{1}{3}(4 \pi G \mu)^{2}<\left(v_{s} \gamma_{s}\right)^{2}>
$$


Also, since $\left(t_{i}, t_{f}\right)=\left(t_{r e c}, t_{0}\right),\left(\Psi_{\min }, \Psi_{\max }\right)$ is given by $(13), Q=23$ (from (15) with $\alpha=1.26$ ) and $N=200 M$. Thus, substituting in (12) we obtain

$$
P_{K S}(k)=4 \times 10^{4}(G \mu)^{2} b \sum_{q=0}^{Q=23} \frac{\sin \left(0.008 \xi \alpha^{q} k\right)}{\alpha^{q} k^{2}}
$$

where $b \equiv M<\left(v_{s} \gamma_{s}\right)^{2}>$.

\section{Potential perturbations:}

(a) Wakes: We are interested in all the wakes present on the last scattering surface thus causing potential perturbations on it. Those wakes were formed at time $t_{i}$, with $t_{e q} \leq t_{i} \leq t_{r e c}$ (since perturbations start to grow at $t_{e q}$ ). All these wakes must be taken into account with their growth factors. In the case of wakes, the sum of equation (12) will run not on the epoch that the photon was affected (as was the case for the Kaiser-Stebbins component of the spectrum) but on the growth factor and the size of the wake present on the last scattering surface. In this case, we have $\left(t_{i}, t_{f}\right)=\left(t_{e q}, t_{r e c}\right)$ which implies $Q=3.6$ and $N=450 M$. Using (5) and following the same steps as for the Kaiser-Stebbins effect we obtain

$$
P_{w}(k)=1.3 \times 10^{2}(G \mu)^{2} b \xi^{4} \sum_{q=0}^{4} \frac{1}{\alpha^{3 q}}\left(\frac{\sin g}{g}-2\left(\frac{\sin g}{g}\right)^{2}\right)^{2}(
$$

where

$$
g=7 \times 10^{-3} \xi k \alpha^{q}
$$

(b) Loops: Using (6), (7) and (12) with $\left(t_{i}, t_{f}\right)=\left(t_{e q}, t_{r e c}\right)$ we obtain

$$
P_{l}(k)=5.6 \beta^{2}(G \mu)^{2} M_{l} \sum_{q=0}^{4} \frac{\sin ^{2}\left(0.014 k \xi_{l} \alpha^{q}\right)}{\alpha^{5 q} k^{2}}
$$

where $M_{l}$ is the cubic root of the number of loops per horizon scale (i.e. the number of loops that intersect a horizon size arc on the sky) and $\xi_{l}$ is typical size of loops as a fraction of the horizon scale. The exact values of the parameters $\beta, \xi_{l}$ and $M_{l}$ are unimportant for our calculations, since it will be seen that for all values of parameters which are consistent with string simulations, 
the term $P_{l}(k)$ is orders of magnitude smaller than the other terms contributing to the power spectrum for $k \leq 10^{4}$ ( $\theta \geq 1$ arcmin). For definiteness we will use $\beta=10, M_{l}=40$ and $\xi_{l}=10^{-3}$ (values which tend to overestimate the contribution of loops). Even with these values it will be seen that loops are unimportant.

3. Doppler effect: Since velocity perturbations on the plasma do not grow before recombination we will only consider the contribution of strings present at the time of recombination. Using (2) and (12) with $t_{i}=$ $t_{f}=t_{r e c}$ we obtain

$$
P_{D}(k)=1.2 \times 10^{4} \lambda^{2}(G \mu)^{2} b \frac{\sin ^{2}(0.016 \xi k)}{k^{2}}
$$

The total spectrum is therefore given by

$$
P(k)=P_{K S}(k)+P_{w}(k)+P_{l}(k)+P_{D}(k)
$$

\section{Parameter Fixing-Scale Invariance}

Before being able to make predictions about ongoing CMB experiments we must determine the only free parameter $G \mu$ as well as the parameters $b, \lambda$ and $\xi$. String simulations (Bennett \& Bouchet 1988; Allen \& Shellard 1990) indicate that $M \simeq 10$ while $\left(v_{s} \gamma_{s}\right)_{r m s} \simeq 0.15-0.2$ implying $b \simeq 0.24$ and $\lambda \simeq$ 6 . We will verify these values by directly fitting our spectrum with partial CMB spectra obtained by simulations on large angular scales. Bouchet, Bennett and Stebbins (hereafter BBS) have used numerical simulations to calculate the term $P_{K S}(k)$ for a single expansion step. Their result for the total power on angular scales smaller than $\theta_{*}$ with $t_{i}=t_{r e c}, t_{f} \simeq 2 t_{r e c}$ is

$$
\begin{aligned}
P_{B B S}\left(\theta \leq \theta_{*}, \Theta_{i}=\Theta_{r e c}\right) & =\int_{2 \pi / \theta_{*}}^{\infty} \frac{d^{2} k}{(2 \pi)^{2}} P_{B B S}(k) \\
& =(6 G \mu)^{2}\left(\frac{\theta_{*}^{1.7}}{0.0012+\theta_{*}^{1.7}}\right)^{0.7}
\end{aligned}
$$

Our analysis, focusing on a line across the sky rather than a patch predicts

$$
P_{a n}\left(\theta \leq \theta_{*}, \Theta_{i}=\Theta_{r e c}\right)=2 \int_{2 \pi / \theta_{*}}^{\infty} \frac{d k}{(2 \pi)} P_{K S}^{Q=0}(k)
$$

In Figure 1 we show $P_{a n}\left(\theta_{*}\right)$ for $b=0.237$ and $\xi=0.45$ (continous line) superimposed on $P_{B B S}\left(\theta_{*}\right)$ (dashed line). We also show the $1 \sigma$ errors to 
$P_{a n}\left(\theta_{*}\right)$ obtained from the variance of $a_{n}^{2}$ (dotted lines). The values $b=0.237$ and $\xi=0.45$ were chosen in order to obtain the best fit to $P_{B B S}$ but they are in very good agreement with the expected values (obtained for $M \simeq 10$, $\left(v_{s} \gamma_{s}\right)_{r m s} \simeq 0.15$ and string radius of curvature about half the horizon scale).

Figure 2a shows a superposition of the components of the spectrum (equations (22), (23), (25) and (26)) with the above choice of parameters. The sums were performed using Mathematica (Wolfram 1991). Clearly the Kaiser-Stebbins term (continous line) dominates on large angular scales $\left(\theta>4^{\circ}\right)$ while the Doppler term (long dashed line) is dominant on smaller scales. The contribution of potential perturbations by wakes (dotted line) is less important but is clearly not negligible especially on scales of a few $\operatorname{arcmin}(k \simeq 1500)$. Finally, the contibution of loops (short dashed line) is negligible on all scales larger than $2-3 \operatorname{arcmin}(k \leq 8000)$. Figure $2 \mathrm{~b}$ shows the product $k P(k)$ fot the total spectrum (equation (27)) with $1 \sigma$ errors denoted by the dotted lines.

One of the most interesting questions that may be addressed using the spectrum of Figure 2b is 'What is the effective power spectrum index $n$, predicted by cosmic strings on COBE angular scales?'. Previous studies (Bennett, Stebbins \& Bouchet 1992; Perivolaropoulos 1993a) have addressed this question without taking into account the effects of potential and Doppler perturbations. The correlation function $C_{1}(\theta)$ for perturbations along a great circle is given in terms of $P(k)$ as

$$
C_{1}(\theta)=<\frac{\delta T}{T}(\phi) \frac{\delta T}{T}(\theta+\phi)>_{\phi}=\frac{1}{(2 \pi)^{2}} \sum_{k=-\infty}^{k=+\infty} P(k) e^{i k \theta}
$$

For $2 \mathrm{~d}$ maps the corresponding equation is $(l \gg 1)$ (Efstathiou 1989)

$$
C_{2}(\theta) \simeq \frac{1}{(2 \pi)^{2}} \int d^{2} l C_{l} e^{i \vec{l} \cdot \vec{\theta}}
$$

By isotropy we must have $C_{1}(\theta)=C_{2}(\theta)$. It may also be shown that $l^{2} C_{l} \sim$ $l^{n-1}$ where $n$ is the power spectrum index. Since both $k$ and $l$ are Fourier conjugate of $\theta$ we have $k \simeq l$. Also (30) and (31) imply that $P(k) \simeq \pi l C_{l}$ and therefore

$$
k P(k) \sim k^{n-1}
$$

Figures $3 \mathrm{a}$ and $3 \mathrm{~b}$ show the best linear fit of the log-log plot $k P(k)$ vs $k$, for $5 \leq k \leq 20$ and $5 \leq k \leq 100$ respectively. The best fits give $n=1.35$ (Figure $3 \mathrm{a}$ ) and $n=1.48$ (Figure $3 \mathrm{~b}$ ). This result indicates that cosmic 
strings favor values of $n$ somewhat larger than 1 in agreement with recent indications from the Tenerife experiment and the second year data of COBE (Bennett et. al. 1994) which favor $n \simeq 1$.5. In contast it is much harder for inflationary models to explain such high values of $n$ (Lyth \& Liddle 1994; Steinhardt 1993).

There is a simple analytic way to show that in the sector of the power spectrum where the Kaiser-Stebbins effect dominates, a scale invariant ( $n \simeq$ 1) spectrum should be expected. For $\tilde{f}_{1}^{\Psi}(k) \sim \Psi \tilde{f}_{1}^{\Psi=1}(k \Psi)$ (as in the case of the Kaiser-Stebbins seed functions), (12) may be writen as

$$
P(k)=\sum_{q=0}^{Q} P_{q}(k)=\sum_{q=0}^{Q} \alpha^{q} P_{0}\left(\alpha^{q} k\right) \simeq \alpha P(\alpha k), \quad \Psi_{\max }^{-1} \leq k \leq \Psi_{\min }^{-1}
$$

Therefore

$$
k P(k) \sim \text { const }
$$

which indicates a scale invariant spectrum for the Kaiser-Stebbins term in the angular scale range $\theta \geq 2^{\circ}\left(k \leq \Psi_{\text {min }}^{-1}\right)$. The Kaiser-Stebbins term plotted in Figure 2a (continous line) is in agreement with this result (the best fit for this component of the spectrum is obtained for $n=1.12$ ).

\section{Predictions-Conclusion}

It is now straightforward to use the derived power spectrum with the appropriate filter functions in order to make predictions about ongoing experiments. The predicted rms temperature fluctuations $\frac{\Delta T}{T} r m s$ for an experiment with window function $W(k)$ is

$$
{\frac{\Delta T}{T_{r m s}}}=(C(0))^{1 / 2}=\left[\frac{1}{2 \pi^{2}} \sum_{k=0}^{\infty} P(k) W(k)\right]^{1 / 2}
$$

For COBE we have $\frac{\Delta T}{T}_{r m s} \simeq 1.1 \times 10^{-5}$ and $W(k) \simeq e^{-k^{2} / 18^{2}}$. Thus (35) may be used to fix the single free parameter $G \mu$. The result is

$$
G \mu \simeq 1.6
$$

in agreement with previous analyses (Bennett, Stebbins \& Bouchet 1992; Perivolaropoulos 1993a) valid on the COBE angular scales $\left(\theta \sim 10^{\circ}\right)$. This result, combined with the fit to the BBS simulation, completely fixes the predicted power spectrum $P(k)$ and the corresponding correlation function 
$C(\theta)$. Figure 4 shows this correlation function smoothed by the COBE filter function, superimposed with the COBE data (Smoot et. al. 1992; Wright et. al. 1992).

Using $W(k)=e^{-\left(k-k_{0}\right)^{2} / \Delta k^{2}}$ and fixing $k_{0}, \Delta k$ for some of the ongoing experiments (TEN: Watson et. al. 1992; SP91: Gaier T. et.al. 1992; SP91: Schuster et. al. 1993; MSAM: Cheng et. al. 1993; SK: Wollack et. al. 1993; MAX: Meinhold et. al. 1993; MAX: Gunderson et. al. 1993; WD: Tucker et. al. 1993; OVRO: Readhead et.al. 1989) we are in position to predict the corresponding value of $\frac{\Delta T}{T} r m s$ thus testing the cosmic string model. These predictions with $1 \sigma$ errors coming from the variance of $a_{n}^{2}$ are shown in Table 1. We also show some of the detections and upper limits existing to date as well as the predictions of inflationary models for $0.8 \leq n \leq 1.0, \Lambda=0$ (Bond et. al. 1994). At this time both inflationary models and cosmic strings appear to be consistent with detections at the $1 \sigma$ level. However, as the quality of observations improves, this may very well change in the near future.

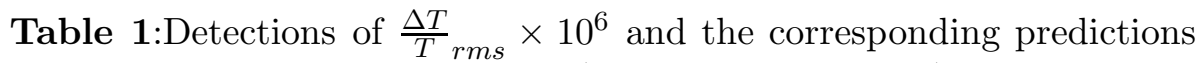
of the string and inflationary models $(0.8 \leq n \leq 1.0, \Lambda=0)$ normalized on COBE.

\begin{tabular}{|c|c|c|c|c|c|}
\hline Experiment & $k_{0}$ & $\Delta k$ & Detection & Strings & Inflation \\
\hline COBE & 0 & 18 & $11 \pm 2$ & $11 \pm 3$ & $11 \pm 2$ \\
\hline TEN & 20 & 16 & $\leq 17$ & $13 \pm 3$ & $9 \pm 1$ \\
\hline SP91 & 80 & 70 & $11 \pm 5$ & $20 \pm 5$ & $12 \pm 2$ \\
\hline SK & 85 & 60 & $14 \pm 5$ & $19 \pm 4$ & $12 \pm 3$ \\
\hline MAX & 180 & 130 & $\leq 30(\mu P e g)$ & $21 \pm 5.5$ & $16 \pm 5$ \\
\hline MAX & 180 & 130 & $49 \pm 8(G U M)$ & $21 \pm 5.5$ & $16 \pm 5$ \\
\hline MSAM & 300 & 200 & $16 \pm 4$ & $19 \pm 4$ & $24 \pm 6$ \\
\hline OVRO22 & 600 & 350 & - & $13 \pm 4$ & $17 \pm 7$ \\
\hline WD & 550 & 400 & $\leq 12$ & $17.5 \pm 4.5$ & $7 \pm 2$ \\
\hline OVRO & 2000 & 1400 & $\leq 24$ & $13.5 \pm 3.5$ & $7 \pm 3$ \\
\hline
\end{tabular}

In conclusion, we have demonstrated, using a simple analytical method, that the CMB spectrum predicted by the cosmic string model can be calculated in a straightforward way including all the relevant sources of perturbations. We have also shown that our results are consistent with numerical simulations even though their validity extends beyond the resolution of present simulations. Finally we showed that the predicted power spectrum index is slightly larger than $1\left(n_{e f f} \simeq 1.4\right)$ and that the predicted rms tem- 
perature fluctuations $\frac{\Delta T}{T} r m s$ are consistent with detections to this date on all angular scales larger than 2-3 arminutes.

Our analysis has assumed standard recombination and values of cosmological parameters $\left(\Omega_{0}=1, h=1 / 2, \mathrm{CDM}, \Lambda=0\right)$. It is important to extend our results to less standard cases including reionization or presence of Hot Dark Matter. Work in this direction is in progress.

\section{Acknowledgements}

I wish to thank R. Brandenberger and R. Moessner for interesting discussions and for providing helpful comments after reading the paper. I also thank Tanmay Vachaspati for stimulating discussions which initiated this project. This work was supported by a CfA Postdoctoral Fellowship.

\section{Figure Captions}

Figure 1:The total power on scale less than $\theta_{*}$ produced by cosmic strings during one expansion step starting at $t_{r e c}$.

Figure 2a: The four components of the power spectrum of CMB perturbations induced by cosmic strings

Figure 2b: The total power spectrum of string perturbations along a great circle on the sky.

Figure 3a: The best linear fit to the total power spectrum for $5 \leq k \leq 20$.

Figure 3b: The best linear fit to the total power spectrum for $5 \leq k \leq 100$.

Figure 4: The cosmic string predicted correlation function smoothed on COBE scales. Superimposed are the first year COBE data. 


\section{References}

Allen B. \& Shellard E. P. S. 1990, Phys.Rev.Lett. 64, 119.

Albrecht A. \& Stebbins A. 1993. Phys. Rev. Lett. 69, 2615.

Bardeen J., Steinhardt P. \& M.Turner M. 1983, Phys.Rev. D28, 679.

Bennett C. L. et. al. 1994, Cosmic Temperature Fluctuations from Two

.... Years of COBE DMR Observations, submitted to Ap. J.

....(ASTROPH-9401012).

Bennett D. \& Bouchet F. 1988, Phys.Rev.Lett. 60, 257.

Bennett D., Stebbins A. \& Bouchet F. 1992, Ap.J.(Lett.) 399, L5.

Bond R., Crittenden R., Davis R., Efstathiou G. \& Steinhardt P. 1994,

....Phys. Rev. Lett. 72, 13.

Bouchet F. R., Bennett D. P. \& Stebbins A. 1988, Nature 335, 410.

Bouchet F. R. 1988, in 'The Formation and Evolution of Cosmic Strings', ....ed. by Gibbons G., Hawking S. \& Vachaspati T. (Cambridge Univ.

....Press), p 359 .

Brandenberger R. 1992, 'Topological Defect Models of Structure Formation

....After the COBE Discovery of CMB Anisotropies', Brown preprint

....BROWN-HET-881 (1992), publ. in proc. of the International School of

....Astrophysics "D.Chalonge", 6-13 Sept.1992, Erice, Italy, ed.

....N.Sanchez (World Scientific, Singapore, 1993).

Brandenberger R., Kaiser N., Shellard E. P. S., Turok N. 1987. Phys.Rev.

....D36, 335.

Brandenberger R. \& Turok N. 1986, Phys. Rev. D33, 2182.

Cheng E. S. et. al. 1993, A Measurement of the Medium Scale Anisotropy

.... in the $C M B$, preprint MSAM-93A.

Coulson D., Ferreira P., Graham P. \& Turok N. 1993, П in the Sky? CMB

....Anisotrtopies from Cosmic Defects, PUP-TH-93-1429,

....hep-ph/9310322.

Efstathiou G. 1989, in 'Physics of the Early Universe', SUSSP 36, 1989, ed. ....J.Peacock, A.Heavens \& A.Davies (IOP Publ., Bristol, 1990).

Gaier T. et.al. 1992, (SP91), Ap. J. Lett. 398, L1.

Gott R. 1985, Ap. J. 288, 422.

Gott J. et. al. 1990, Ap.J. 352, 1.

Guth A. \& Pi S. -Y. 1982, Phys.Rev.Lett. 49, 110.

Gunderson J. et. al. 1993, (MAX), Ap. J. Lett. 413, L1.

Hara T. \& Miyoshi S. 1993, Ap. J. 405, 419.

Hawking S. 1982, Phys.Lett. 115B, 295. 
Hindmarsh M. 1993, Small Scale CMB Fluctuations from Cosmic Strings, ....DAMTP-93-17, astro-ph 9307040.

Kaiser N. \& Stebbins A. 1984, Nature 310, 391.

Kibble T. W. B. 1976, J.Phys. A9, 1387.

Luo X. 1993, The Angular Bispectrum of the CMB, CFPA-93-TH-42, .... astro-ph 9312004.

Lyth D. \& Liddle A. 1994,Observational Constraints on the Spectral Index, ....Contribution to the 1993 Capri CMB Workshop. SUSSEX-AST

....93/12-1, astro-ph/9401014

Meinhold P. et. al. 1993, (MAX), Ap. J. Lett. 409, L1.

Moessner R., Perivolaropoulos L. \& Brandenberger R. 1993, A Cosmic

....String Specific Signature on the CMB, Ap. J. in press,

.... astro-ph/9310001.

Perivolaropoulos L. 1993a, Phys.Lett. B298, 305.

Perivolaropoulos L. 1993b, Phys. Rev. D48, 1530.

Perivolaropoulos L. 1993c, The Fourier Space Statistics of Seed-like

....Cosmological Perturbations, M.N.R.A.S. in press, CfA-3591,

.... astro-ph/9309023.

Perivolaropoulos L. \& Vachaspati T. 1993,Peculiar Velocities and

....Microwave Background Anisotropies from Cosmic Strings, Ap. J. Lett.

....in press, CfA-3590, astro-ph/9303242.

Perivolaropoulos L., Brandenberger R. \& Stebbins A. 1990, Phys.Rev.

....D41, 1764.

Readhead A.C.S. et.al. 1989, (OVRO), Ap. J. 346, 556.

Sachs R. \& Wolfe A. 1967, Ap. J. 147, 73.

Schuster J. et. al. 1993, (SP91), Ap. J. Lett. 412, L47.

Smoot G. et. al. 1992, (COBE), Ap. J. Lett. 396, L1.

Starobinsky A. 1982, Phys.Lett. 117B, 175.

Stebbins A. et. al. 1987, Ap. J. 322, 1.

Stebbins A. 1988, Ap.J. 327, 584.

Stebbins A. 1993, Ann. N.Y. Acad. Sci. 688 (Texas/PASCOS

Proceedings), 824.

Steinhardt P. 1993, private communication.

Traschen J., Turok N. \& Brandenberger R. 1986, Phys. Rev. D34, 919.

Tucker G. S. et. al. 1993, (WD), Princeton preprint.

Turok N. and Brandenberger R. 1986, Phys. Rev. D33, 2175.

Vachaspati T. 1986, Phys. Rev. Lett. 57, 1655.

Vachaspati T. 1992a, Phys.Lett. B282, 305.

Vachaspati T. 1992b, Phys. Rev. D45, 3487. 
Vachaspati T. \& Vilenkin A. 1991. Phys. Rev. Lett. 67, 1057-1061.

Veeraraghavan S. \& Stebbins A. 1990, Ap.J. 365, 37.

Vilenkin A. 1981, Phys.Rev. D23, 852.

Vilenkin A. 1985, Phys.Rep. 121, 263.

Vollick D. N. 1992, Phys. Rev. D45, 1884.

Watson R. A. et. al. 1992, (TEN), Nature 357, 660.

Wright E. L. et. al. 1992, Ap. J. Lett. 396, L5.

Wolfram S. 1991, Mathematica version 2.0, Addison-Wesley.

Wollack E. J. et. al. 1993, (SK), Ap. J. Lett. 419, L49. 
This figure "fig1-1.png" is available in "png" format from: http://arxiv.org/ps/astro-ph/9402024v1 
This figure "fig2-1.png" is available in "png" format from: http://arxiv.org/ps/astro-ph/9402024v1 
This figure "fig1-2.png" is available in "png" format from: http://arxiv.org/ps/astro-ph/9402024v1 
This figure "fig2-2.png" is available in "png" format from: http://arxiv.org/ps/astro-ph/9402024v1 
This figure "fig1-3.png" is available in "png" format from: http://arxiv.org/ps/astro-ph/9402024v1 
This figure "fig2-3.png" is available in "png" format from: http://arxiv.org/ps/astro-ph/9402024v1 